BULL. AUSTRAL. MATH. SOC.

VOL. 19 (1978), 67-75.

\title{
A note on a paper of E.R. Love
}

\section{F. Fehér}

\begin{abstract}
Applying a new and very elegant method of proof of the Schur-Hardy inequality, given by E.R. Love at the Oberwolfach conference on Linear Spaces and Approximation (1977), norm estimates of integral operators with homogeneous kernels are established in the setting of abstract function norms. Applications to Flett's inequality, to integral means, and to fractional integrals are given.
\end{abstract}

Let $\rho$ denote a function norm on the set $P((0, \infty))$ of all nonnegative, Lebesgue measurable functions on $(0, \infty)$, that is, a mapping $\rho: P((0, \infty)) \rightarrow[0, \infty]$ with the properties that, for all $f, g \in P$,

(i) $\rho(f)=0$ if and only if $f=0$ almost everywhere, $\rho(\lambda f)=\lambda \rho(f) \quad(\lambda>0) ; \quad \rho(f+g) \leq \rho(f)+\rho(g) ;$

(ii) $f \leq g$ almost everywhere only if $\rho(f) \leq \rho(g)$.

The space $L^{\rho}$ of all measurable, real valued functions $f$ with $\|f\|_{\rho}:=\rho(|f|)<\infty$, functions which coincide almost everywhere being identified, is called the normed Kothe space generated by $\rho$, see [9, p. 42]. The operator norm on $L^{\rho}$ of the dilation operator $E_{s}$, defined by $\left(E_{s} f\right)(t)=f(s t), s, t>0$, is called the indicator function $h(s)$ of $L^{\rho}$, that is, $h(s):=\left\|E_{s}\right\|_{\left[L^{\rho}\right]}$. If $\|\cdot\|_{\rho}$ is, in particular, the Lebesgue norm $\|\cdot\|_{q}, 1 \leq q<\infty$, then $h(s)=s^{-1 / q}$.

In the sequel let $K(t, s)$ denote a nonnegative, measurable function

Received 20 June 1978. Communicated by E.R. Love. 
of $t, s>0$, and let $K$ be the operator defined by

$$
(K f)(t)=\int_{0}^{\infty} K(t, s) f(s) d s \quad\left(t>0, f \in L^{\rho}\right) .
$$

THEOREM 1. Let $K(t, s)$ be homogeneous of degree $\gamma$, where $\gamma \geq-1$, such that

$$
A:=\int_{0}^{\infty} K(1, s) s^{-(1+\gamma)} h(s) d s<\infty
$$

and $(\cdot)^{I+\gamma} f \in L^{\rho}$. Then, if $L^{\rho}$ is complete, $K f \in L^{\rho}$, and

$$
\|K f\|_{\rho} \leq A\left\|(\cdot)^{1+\gamma_{f}}\right\|_{\rho}
$$

Proof. By the homogeneity of the kernel $K(t, s)$ one has, with $s=t u$,

$$
\begin{aligned}
|(K f)(t)| & \leq \int_{0}^{\infty} K(t, t u)|f(t u)| t d u \\
& =\int_{0}^{\infty} K(1, u) t^{1+\gamma}\left|\left(E_{u} f\right)(t)\right| d u \\
& =\int_{0}^{\infty} K(I, u) u^{-(1+\gamma)}\left|\left[E_{u}(\cdot)^{1+\gamma} f\right)(t)\right| d u .
\end{aligned}
$$

Now (2) follows on account of the monotonicity of $\rho$ and the completeness of $L^{\rho}$.

This is essentially the method of proof of Love [8, Theorem 1.2]. That theorem follows from our Theorem 1 by taking $\gamma=-1$ and $\|\cdot\|_{\rho}=\|\cdot\|_{q}, \quad 1 \leq q<\infty$, (the Lebesgue norm), namely

COROLLARY 2 (Schur-Hardy inequality). Let $K(t, s)$ be homogeneous of degree -1 such that

$$
A_{q}=\int_{0}^{\infty} K(1, s) s^{-1 / q} d s<\infty
$$

and $f \in L_{q}$. Then $K f \in L_{q}$ and

$$
\|K f\|_{q} \leq A_{q}\|f\|_{q} .
$$


A slightly modified version of Theorem $l$ is possible if one proceeds as follows: if $f^{*}$ denotes the nonincreasing rearrangement of $f$ (for definition, see for example [4]), and $f^{* *}(t):=(1 / t) \int_{0}^{t} f^{*}(s) d s$, then $f^{*}(t) \leq f^{* *}(t)$, and $\left(E_{\mathcal{u}} f\right)^{*}(t)=\left(E_{\mathcal{u}} f^{*}\right)(t)$. Therefore

$$
\begin{aligned}
|(K f)(t)| & \leq t^{1+\gamma} \int_{0}^{\infty} K(1, u)\left|\left(E_{u} f\right)(t)\right| d u \\
& \leq t^{1+\gamma} \int_{0}^{\infty} K^{*}(I, u)\left(E_{u} f^{*}\right)(t) d u \\
& =\int_{0}^{\infty} K^{*}(1, u) u^{-(1+\gamma)}\left(E_{u}(\cdot)^{1+\gamma} f^{*}\right)(t) d u,
\end{aligned}
$$

yielding

THEOREM $1^{*}$. If $K(t, s)$ is homogeneous of degree $\gamma$, where $\gamma \geq-1$, such that

$$
A^{*}:=\int_{0}^{\infty} K^{*}(1, s) s^{-(1+\gamma)} h(s) d s<\infty,
$$

and $f$ a measurable function on $(0, \infty)$ with $(\cdot)^{1+\gamma_{f^{* *}} \in L^{\rho}}$, then $K f \in L^{\rho}$ and

$$
\|K f\|_{\rho} \leq A^{*}\left\|(\cdot)^{1+\gamma} f^{* *}\right\|_{\rho} .
$$

Note that Theorem $I^{*}$ as well as Theorem $l$ include in particular the cases when $\|\cdot\|_{\rho}$ is the Lebesgue, Lorentz, or Orlicz norm, respectively. In the Lebesgue case, for instance, Theorem $l^{*}$ reduces to

COROLLARY $2^{*}$. Let $\gamma \geq-1,1 \leq q<\infty$, and $p:=q /(1+q(1+\gamma))$. If $K(t, s)$ is homogeneous of degree $\gamma$ such that

$$
A_{q}^{*}:=\int_{0}^{\infty} K^{*}(1, s) s^{-(1+\gamma)} s^{-1 / q} d s<\infty,
$$

and $f \in L_{p q}$ (equals the Lorentz space, see [4]), then $K f \in L_{q}$ and

$$
\|K f\|_{q} \leq(p / q)^{1 / q} A_{q}^{*}\|f\|_{p q}
$$


Evidently Corollary 2* implies [8, Theorem 2.1] by replacing $\gamma$ by $-r^{-1}$, and observing that $\|\cdot\|_{p q} \leq(p /(p-1))\|\cdot\|_{p}$ for $1<p \leq q$. Our constant $A_{q}^{*}$ is different from that of [8], since on the right hand side of $\left(3^{*}\right)$ the Lorentz norm is used instead of the Lebesgue norm. A simple computation shows, however, that the constant $C$ of [8] can be estimated by $C \leq A_{q}^{*} / r$. Indeed (note that $r \geq 1$ ):

$$
\begin{aligned}
c: \equiv\left\|K(1, s) s^{-1 / q_{1}}\right\|_{r} & \leq\left\|K^{*}(1, s) s^{-1 / q_{1}}\right\|_{r}=\| K^{*}(1, s) s^{-1 / q_{1 p}^{*}} \\
& \leq \| K^{*}(1, s) s^{-1 / q_{\|^{*}}^{*}}=(1 / r) A_{q}^{*},
\end{aligned}
$$

where $\|\cdot\|_{p q}^{*}$ denotes the modified Lorentz norm with $f^{* *}$ replaced by $f^{*}$.

REMARK. It is well known that the $\underline{\underline{K}}$-functional of Peetre with respect to the spaces $L_{1}$ and $L_{\infty}$, namely

$$
\underline{\underline{K}}\left(t, f ; L_{1}, L_{\infty}\right):=\inf \left\{\left\|f_{1}\right\|_{1}+t\left\|f_{2}\right\|_{\infty}: f=f_{1}+f_{2}, f_{1} \in L_{1}, f_{2} \in L_{\infty}\right\},
$$

can be expressed in terms of $f^{*}$. Indeed,

$$
\underline{\underline{K}}\left(t, f ; L_{1}, L_{\infty}\right)=\int_{0}^{t} f^{*}(s) d s=t f^{* *}(t) .
$$

Therefore the assertion of Theorem $1^{*}$ remains valid if $\left(2^{*}\right)$ is replaced by

$$
\|K f\|_{\rho} \leq A^{*}\left\|t \underline{\underline{K}}\left(t, f ; L_{1}, L_{\infty}\right)\right\|_{\rho} .
$$

With the notations of [5], in particular $\gamma=-\theta, \theta \leq 1$, and

$$
\|f\|_{\theta, \rho ; \underline{\underline{K}}}:=\rho\left(t^{-\theta} \underline{\underline{\underline{K}}}\left(t, f ; L_{1}, L_{\infty}\right)\right) \text {, }
$$

Theorem $1^{*}$ can be reformulated in the language of interpolation theory as follows.

COROLLARY $3^{*}$. Under the assumptions of Theorem $1^{*}$ the operator $K$ is a bounded operator from the interpolation space $\left(L_{1}, L_{\infty}\right)_{-\gamma, \rho ; \underline{K}}$ of $L_{1}$ and $L_{\infty}$ into the space $L^{\rho}$ such that (4*) holds.

Here recall that 


$$
\left(L_{1}, L_{\infty}\right)_{-\gamma, \rho ; \underline{\underline{K}}}:=\left\{f \in L_{1}+L_{\infty}: t^{\gamma} \underline{\underline{K}}\left(t, f ; L_{1}, L_{\infty}\right) \in L^{\rho}\right\} \text {. }
$$

Finally let us discuss some applications of the above theorems to special kernels. As a first example, consider the average operator $P_{\theta}$, $\theta>0$ (see Boyd [2]), defined by

$$
\left(P_{\theta} f\right)(t):=t^{-\theta} \int_{0}^{t} s^{\theta-1} f(s) d s \quad(t>0) .
$$

This operator is obviously of type (1) with kernel

$$
K(t, s)=t^{-\theta} s^{\theta-1} \chi_{(0, t)}(s),
$$

$X_{(0, t)}$ denoting the characteristic function of the interval $(0, t)$. Since this kernel is homogeneous of degree $\gamma=-1$, Theorem 1 yields that

$$
\left\|P_{\theta} f\right\|_{\rho} \leq A_{\theta}\|f\|_{\rho} \quad\left(f \in L^{\rho}\right),
$$

if

$$
A_{\theta}:=\int_{0}^{1} s^{\theta-1} h(s) d s<\infty .
$$

In particular, if $\theta=1$ and $\|\cdot\|_{p}=\|\cdot\|_{q}, q>1$, then (5) reduces to the classical Hardy inequality (see [7, p. 240] or [3] and the literature quoted there). For the case of Lorentz norms see also [10]. More generally, if $\|\cdot\|_{\rho}$ is a rearrangement-invariant norm - for definition see, for example, [2], [5] - then (5) is precisely the generalized Hardy inequality in the setting of rearrangement-invariant norms, as established in Butzer and Fehér [3]. Let us also mention that the constant $A_{\theta}$ of (6) is identical with that of [3]; therefore (6) is equivalent to the index condition $\alpha<\theta$, where $\alpha$ denotes the upper index of $\rho$, that is to say,

$$
\alpha=\inf _{0<s<1}(-\log h(s) / \log s)
$$

(compare [5]).

As a second example one might consider the modified operator of fractional integration ${ }_{\lambda}, \lambda>0$, defined by (see [8]) 


$$
\left(M_{\lambda} f\right)(t):=t^{-\lambda} \int_{0}^{t} \frac{(t-s)^{\lambda-1}}{\Gamma(\lambda)} f(s) d s \quad(t>0) .
$$

As the kernel of this operator is again homogeneous of degree -1, Theorems 1 and $1^{*}$, as well as the above corollaries, immediately apply to this operator.

$$
\text { If, in particular, }\|\cdot\|_{\rho}=\|\cdot\|_{p q}, I \leq p, q<\infty \text {, then } h(s)=s^{-1 / p}
$$

and Theorem 1 , for example, asserts that for $p>1$,

$$
\left\|{ }_{\lambda} f\right\|_{p q} \leq \frac{\Gamma(1-1 / p)}{\Gamma(1-1 / p+\lambda)}\|f\|_{p q} \quad\left(f \in L_{p q}\right) .
$$

Similarly, Elett's inequality [6], namely,

$$
\left\|(\cdot)^{-\beta-1 / q_{M} f}\right\|_{q} \leq \text { const. }\left\|(\cdot)^{-\beta-1 / p_{f}}\right\|_{p}(\beta>-1),
$$

is easily obtained from Corollary $2^{*}$ (if $A_{q}^{*}<\infty, p>1$ ) by taking

$$
K(t, s)=\chi_{(0, t)}(s) t^{-\lambda-\beta-1 / q}(t-s)^{\lambda-1} s^{\beta+I / P} / \Gamma(\lambda)
$$

and replacing $f(s)$ by $s^{-\beta-1 / p} f(s)$ (see [8]).

If one chooses $\|\cdot\|_{p}=\|\cdot\|_{p q}$, then Corollary $2^{*}$ implies a generalized version of Flett's inequality with respect to Lorentz norms (compare [11]).

A very important example of an integral operator the kernel of which is homogeneous of degree $\gamma>-I$ is the operator $I_{\lambda}$ of fractional integration of order $\lambda>0$, in the sense of Riemann-Liouville, defined by

$$
\left(I_{\lambda} f\right)(t):=\frac{1}{\Gamma(\lambda)} \int_{0}^{t}(t-s)^{\lambda-I} f(s) d s \quad(t>0) .
$$

Since the kernel of $I_{\lambda}$ is homogeneous of degree $\gamma=\lambda-1$, Corollary $2^{*}$ yields

COROLLARY $4 *$. Assume that $1<q<\infty, 0<\lambda<1-1 / q$, and $p:=q /(1+\lambda q)$. If $f \in L_{p}$, then $I_{\lambda} f \in L_{q}$ and

$$
\left\|I_{\lambda} f\right\|_{q} \leq \frac{p}{p-1} \frac{\Gamma(1-\lambda-1 / q)}{\Gamma(1-1 / q)}\|f\|_{p} .
$$

This corollary is in accordance with [7, Theorem 383]. The proof in 
[7] does not furnish any value of the constant in (9), although rather deep results are used in the proof. In contrast to the very elegant method of proof of Love, the usual way of establishing (9) makes appeal to interpolation theory (in particular to the Marcinkievicz interpolation theorem), a fact which now seems understandable, if one recalls Corollary $3^{*}$.

In case of Lorentz norms there exist quite different proofs for the boundedness of the operator $I_{\lambda}$ (compare [13]) using theorems of multiplication and of convolution for Lorentz norms. This boundedness result, namely (compare [13, Proposition 4 with $n=1]$ )

$$
\|(\cdot)^{-\beta_{I_{\lambda}} f \|_{p q}} \leq \text { const. }\left\|(\cdot)^{\alpha} f\right\|_{p_{0} q_{0}},
$$

with $1 / p_{0}=1 / p+\lambda-(\alpha+\beta)$ and $q_{0} \leq q$, can be deduced from theorem 1 by taking $\|\cdot\|_{\rho}=\|\cdot\|_{p q}, K$ the operator (1) with kernel $K(t, s)=\chi_{(0, t)}(s) t^{-\beta}(t-s)^{\lambda-1} s^{\beta} / \Gamma(\lambda)$, and replacing $f(s)$ by $s^{-\beta} f(s)$. Indeed, the left side of (10) is equal to

$$
\begin{aligned}
\left\|(\cdot)^{-\beta} I_{\lambda} f\right\|_{p q} & =\left\|K\left((\cdot)^{-\beta} f\right)\right\|_{p q} \leq A\left\|(\cdot)^{\lambda-\beta} f\right\|_{p q}= \\
& =A\left\|(\cdot)^{1 / p_{0}-1 / p}(\cdot)^{\alpha} f\right\|_{p q} \leq A B\left\|(\cdot)^{\alpha} f\right\|_{p_{0} q} \leq A B \frac{p_{0}}{p_{0}^{-1}}\left\|(\cdot)^{\alpha} f\right\|_{p_{0} q_{0}},
\end{aligned}
$$

with $A=\Gamma(1-\lambda-1 / q+\beta) / \Gamma(1-1 / q+\beta)$. Here use was made of the multiplication theorem for Lorentz norms (compare [1]), namely

$$
\|f g\|_{p q} \leq B\|f\|_{p_{0} q}\|g\|_{p_{1} \infty}
$$

where $1 / p=1 / p_{0}+1 / p_{1}$, and $B$ is a suitable constant, as for example $B=\left(p / p_{0}\right)^{1 / p_{2} 1 / p} p /(p-1)$. For the particular case $p=q$ of $(10)$, that is the Lebesgue case, see for example [12]. 


\section{References}

[1] Pedro Jorge Aranda et Enrique Paule Cattáneo, "Sur les espaces de Lorentz avec poids", C.R. Acad. Sci. Paris Sér. A 264 (1967), 109-112.

[2] David W. Boyd, "Indices of function spaces and their relationship to interpolation", Canad. J. Math. 21 (1969), 1245-1254.

[3] P.L. Butzer and F. Fehér, "Generalized Hardy and Hardy-Littlewood inequalities in rearrangement-invariant spaces", Comment. Math. Prace Mat. (to appear).

[4] Mischa Cotlar and Roberto Cignoli, An introduction to functional anatysis (translated by A. Torchinsky and A. González villalobos. North-Holland, Amsterdam, London; American Elsevier, New York, 1974 ).

[5] F. Fehér, "Interpolation und Indexbedingungen auf rearrangementinvarianten Funktionenräumen. I. Grundlagen und die K-Methode", J. Functional Analysis 25 (1977), 147-161.

[6] T.M. Flett, "A note on some inequalities", Proc. Glasgow Math. Assoc. $4(1.959-60), 7-15$.

[7] G.H. Hardy, J.E. Littlewood, G. Pólya, Inequalities, and edition (Cambridge University Press, Cambridge, 1952; reprinted 1959).

[8] Eric Russel I Love, "Some inequalities for fractional integrals", Linear spaces and approximation (Proc. Conf. Mathematical Research Institute, Oberwolfach, 1977, 177-184. International Series of Numerical Mathematics, 40. Birkhäuser Verlag, Basel, Stuttgart, 1978).

[9] W.A.J. Luxemburg and A.C. Zaanen, Riesz spaces, volume I (NorthHolland, Amsterdam, London, 1971).

[10] P.G. Rooney, "A generalization of some theorems of Hardy", Trans. Roy. Soc. Canada Sect. III (3) 49 (1955), 59-66.

[11] P.G. Roney, "On some properties of certain fractional integrals", Trans. Roy. Soc. Canada Sect. III (3) 50 (1956), 61-70. 
[12] Robert S. Strichartz, "L estimates for integral transforms", Trans. Amer. Math. Soc. 136 (1969), 33-50.

[13] T. Walsh, "On $L^{p}$ estimates for integral transforms", Trans. Amer. Math. Soc. 155 (1971), 195-215.

Lehrstuhl A für Mathematik,

Rheinisch-Westfäl ische Technische Hochschule Aachen,

Aachen,

Germany. 